\title{
Effect of a novel nutraceutical combination on serum lipoprotein functional profile and circulating PCSK9
}

This article was published in the following Dove Press journal:

Therapeutics and Clinical Risk Management

\author{
Maria Pia Adorni' \\ Nicola Ferri² \\ Silvia Marchianò ${ }^{3}$ \\ Valentina Trimarco 4 \\ Francesco Rozza ${ }^{5}$ \\ Raffaele Izzo 5 \\ Franco Bernini ${ }^{1}$ \\ Francesca Zimetti ${ }^{1}$ \\ 'Department of Food and Drug, \\ University of Parma, Parma, \\ ${ }^{2}$ Department of Pharmaceutical and \\ Pharmacological Sciences, University \\ of Padua, Padua, ${ }^{3}$ Department of \\ Pharmacological and Biomolecular \\ Sciences, University of Milan, Milan, \\ ${ }^{4}$ Department of Neurosciences, \\ ${ }^{5}$ Hypertension Research Center, \\ Federico II University, Naples, Italy
}

Background: A beneficial effect on cardiovascular risk may be obtained by improving lipid-related serum lipoprotein functions such as high-density lipoproteins (HDLs) cholesterol efflux capacity (CEC) and serum cholesterol loading capacity (CLC) and by reducing proprotein convertase subtilisin kexin type 9 (PCSK9), independently of lipoprotein concentrations.

Aim: We aimed to evaluate the effect of an innovative nutraceutical (NUT) combination containing red yeast rice (monacolin K $3.3 \mathrm{mg}$ ), berberine $531.25 \mathrm{mg}$ and leaf extract of Morus alba $200 \mathrm{mg}\left(\right.$ LopiGLIK $^{\circledR}$ ), on HDL-CEC, serum CLC and on circulating PCSK9 levels.

Materials and methods: Twenty three dyslipidemic subjects were treated for 4 weeks with the above NUT combination. HDL-CEC was measured using specific cell-based radioisotopic assays; serum CLC and PCSK9 concentrations were measured fluorimetrically and by enzymelinked immunosorbent assay, respectively.

Results: The NUT combination significantly reduced plasma level of the total cholesterol and low-density lipoprotein cholesterol ( $-9.8 \%$ and $-12.6 \%$, respectively). Despite no changes in HDL-cholesterol, the NUT combination improved total HDL-CEC in $83 \%$ of the patients, by an average of $16 \%$, as a consequence of the increase mainly of the ATP-binding cassette A1-mediated CEC (+28.5\%). The NUT combination significantly reduced serum CLC $(-11.4 \%)$ while it did not change PCSK9 plasma levels $(312.9 \pm 69.4 \mathrm{ng} / \mathrm{mL}$ vs $334.8 \pm 103.5 \mathrm{mg} / \mathrm{L}$, before and after treatment, respectively).

Conclusion: The present NUT combination improves the serum lipoprotein functional profile providing complementary beneficial effects, without any detrimental increase of PCSK9 plasma levels.

Keywords: nutraceutical, monacolin K, berberine, Morus alba, HDL, cholesterol efflux capacity, macrophage, PCSK9

\section{Introduction}

In moderate hypercholesterolemia, an available alternative to pharmacological therapy is the use of innovative nutritional compounds, the so-called nutraceuticals (NUTs), as the recent guidelines recommend. ${ }^{1}$ Several NUTs have shown significant effects in improving plasma lipid profile, by reducing total cholesterol (TC) and low-density lipoprotein cholesterol (LDL-C) and, in some cases, by moderately increasing highdensity lipoprotein cholesterol (HDL-C), with a potential positive impact on cardiovascular $(\mathrm{CV})$ risk. $^{2,3}$

However, the mere improvement of quantitative lipid profile may not be the only target to obtain optimal CV benefits, as exemplified by the failure of the hypothesis that HDL-C raising therapy would reduce $\mathrm{CV}$ risk. ${ }^{4}$ Therefore, recent research on HDL
Correspondence: Franco Bernin Department of Food and Drug, University of Parma, Parco Area delle Scienze 27/A, 43I24 Parma, Italy

$\mathrm{Tel}+39521905039$

Fax +39521905040

Email fbernini@unipr.it 
has moved to the evaluation of their functional properties, in particular their ability to remove excess cholesterol from macrophages of the artery wall, by promoting the process of cholesterol efflux to HDL. HDL cholesterol efflux capacity (CEC) has been inversely associated with the prevalence of coronary heart disease ${ }^{5}$ and the incidence of CV events. ${ }^{6,7}$ In addition, it displayed the potential to improve risk stratification beyond the traditional factors, including plasma HDL-C concentrations. ${ }^{8}$

Although HDL-CEC may explain the link between HDL function and atherosclerosis, it only reflects the movement of cholesterol out of cells, while lipid trafficking is bidirectional. ${ }^{9}$ Serum capacity to promote cholesterol uptake and accumulation in macrophages (cholesterol loading capacity [CLC]) might represent, in this context, an additional functional lipid-related parameter that takes into account the movement of cholesterol from lipoproteins into the cells. ${ }^{9}$ Such ability relies on the contribution of all lipoproteins present in the serum, in terms of both concentration and function, and it can be considered an index of the serum pro-atherogenic potential. In support of this idea, our research team and others have previously demonstrated that serum CLC is increased in pathological conditions associated to increased CV risk. ${ }^{9-11}$

An additional identified player of cholesterol homeostasis maintenance is the proprotein convertase subtilisin kexin type 9 (PCSK9), able to degrade the hepatic LDL receptor, resulting in elevated plasma LDL-C concentrations. ${ }^{12}$ Nevertheless, the activity of PCSK9 goes beyond LDL-C modulation and it involves other aspects of cholesterol homeostasis relevant for the pathogenesis of atherosclerosis. ${ }^{12,13}$ The results of a cohort study indicate, in fact, that circulating levels of PCSK9 are associated with $\mathrm{CV}$ events, ${ }^{14}$ atherosclerosis progression, ${ }^{15}$ arterial stiffness, ${ }^{16}$ independently of plasma LDL-C concentrations. Among the possible direct pro-atherogenic effects of PCSK9, there is the inhibition of the cholesterol efflux process promoted by the transporter ATP-binding cassette A1 (ABCA1) in macrophages, as we recently demonstrated. ${ }^{17}$ Based on these observations, the increase of HDL-CEC, the inhibition of serum CLC and the reduction of PCSK9 plasma levels may be a multi-target strategy to counteract cholesterol accumulation in macrophage and foam cell formation.

Given the relevance of the above parameters with respect to $\mathrm{CV}$ risk, ${ }^{18,19}$ as well as the poor data available on the impact of nutritional approaches on these lipoprotein functional properties and, even less, on plasma PCSK9 levels, ${ }^{20-24}$ the aim of the present work was to evaluate the effect of a novel NUT combination ${ }^{25}$ on HDL-CEC, serum CLC and circulating PCSK9 levels.

\section{Materials and methods}

\section{Patients}

LopiWEB is a randomized study (NCT02898805), expecting to enroll $>600$ subjects by physicians, designed to assess the efficacy of LopiGLIK ${ }^{\circledR}$ (Akademy Pharma, Milano, Italy) in real-life. Several regions of Italy are involved in the study and data are collected via electronic case report forms on the website www.lopiweb.it.

In this work, we studied a subgroup population of 23 subjects. The inclusion criteria were age between 18 and 70 years, the presence of metabolic syndrome or moderate hypercholesterolemia not requiring immediate statin treatment or statin intolerance. Pregnant, breastfeeding women and subjects taking hypolipidemic drugs were excluded from the study. The selected patients were not subjected to any kind of therapy before the NUT combination treatment. For this study, subjects received one pill/day containing a NUT combination of $625 \mathrm{mg}$ of Berberis aristata cortex dry extract (corresponding to $531.25 \mathrm{mg}$ of berberine), $220 \mathrm{mg}$ of red yeast rice powder (corresponding to $3.3 \mathrm{mg}$ of monacolin $\mathrm{K}$ ) and $200 \mathrm{mg}$ of Morus alba leaf dry extract (containing $4 \mathrm{mg}$ of 1-deoxynojirimycin) for 16 weeks. During the study, blood withdrawal and serum isolation were planned at baseline and after 4 and 16 weeks of treatment. Our results refer to serum analyses at baseline (T1) and after 4 weeks (T2) of treatment.

Serum samples were collected at the University of Naples after obtaining written informed consent from participants and immediately stored at $-80^{\circ} \mathrm{C}$. Serum lipid profile was assessed by standard laboratory methods. The study was conducted in accordance with the guidelines of the Declaration of Helsinki and the study protocol was approved by the Ethics Committee of the Federico II University of Naples.

\section{HDL-CEC}

HDL-CEC was evaluated through a widely validated isotopic technique. In particular, we adopted different cellular models expressing the most important transporters involved in the promotion of cholesterol efflux to HDL: J774 murine macrophages in basal conditions were taken as a model of aqueous diffusion (AD); J774 macrophages incubated with $0.3 \mathrm{mM}$ of a cAMP analog (8-(p-chlorophenylthio)-cAMP; Sigma-Aldrich Co, St Louis, MO, USA) to induce ABCA1 expression, were used as a model of total HDL-CEC as previously described. ${ }^{5,26}$ The ABCA1 contribution was measured as the difference between total HDL-CEC and AD-CEC. ${ }^{27}$ Chinese hamster ovary cells transfected or not transfected with the human $A B C G 1$ gene were used to 
evaluate ABCG1-mediated CEC. The specific ABCG1 contribution was calculated as the difference between CEC in ABCG1-transfected and not transfected cells. ${ }^{11}$ In all assays, the cells were labeled with $\left[1,2-{ }^{3} \mathrm{H}\right]$-cholesterol (PerkinElmer Inc, Waltham, MA, USA) for 24 hours in the presence of an inhibitor of the cholesterol esterifying enzyme, acylCoA:cholesterol acyltransferase, to ensure all cholesterol was present in the unesterified form. After an equilibration period in medium containing $0.2 \%$ bovine serum albumin (Sigma-Aldrich), the cells were exposed for 4 or 6 hours to $1 \%$ or $2 \%(\mathrm{v} / \mathrm{v})$ (depending on the efflux pathway to be analyzed) of serum HDL fraction from patients before and after NUT combination treatment. HDLs were isolated from whole serum by precipitation of the apolipoprotein B (apoB) containing lipoproteins with polyethylene glycol as previously described $.^{28} \mathrm{HDL}-\mathrm{CEC}$ was expressed as a percentage of the radioactivity released into the medium over the total radioactivity incorporated by the cells. A pool of human normolipidemic sera was tested in each assay as reference standard 1 and its CEC was used to normalize the patient samples CEC values from the different experiments $(n=3)$, to correct for the inter-assay variability. Each experiment was performed in triplicate.

A second pool of human normolipidemic sera as reference standard 2 was tested in each assay and its CEC was the index of the inter-assay variability.

\section{Serum CLC}

THP-1-derived human macrophages were cultured in 24-well plates in the presence of $50 \mathrm{ng} / \mathrm{mL}$ of phorbol 12-myristate 13-acetate (Sigma-Aldrich) for 72 hours to allow the cells to differentiate into macrophages. Cells were exposed for 24 hours to serum collected before and after 4 weeks of treatment with the NUT combination. Whole serum dilution in this model was $10 \%(\mathrm{v} / \mathrm{v})$. Cell cholesterol content was measured by fluorometric detection in cell lysates. ${ }^{10}$ An aliquot of the cell lysates was used to measure cell protein by the modified colorimetric Lowry method. ${ }^{29}$ CLC was defined as macrophage cholesterol content in the cell lysates after exposure of cells to serum and was expressed as micrograms of cholesterol per milligrams of protein.

\section{Determination of apolipoprotein A (apoA)-I sera levels}

To evaluate apoA-I concentration in the HDL fraction, sera were depleted of the apoB containing lipoproteins by precipitation with polyethylene glycol. ${ }^{28}$ apoA-I was measured by enzyme-linked immunosorbent assay (ELISA;
Mabtech, Sweden) following the manufacturer's instructions. The sensitivity ranged from 0.63 to $40 \mathrm{ng} / \mathrm{mL}$ with a minimum detectable concentration of $0.2 \mathrm{ng} / \mathrm{mL}$.

\section{Circulating levels of PCSK9}

Sera PCSK9 levels were measured by ELISA (R\&D Systems, Minneapolis, MN, USA) as previously described. ${ }^{30}$ The sensitivity ranged from 0.030 to $0.219 \mathrm{ng} / \mathrm{mL}$ with a minimum detectable concentration of $0.096 \mathrm{ng} / \mathrm{mL}$.

\section{Statistical analysis}

The sample size was calculated a priori by using the $\mathrm{G}^{*}$ Power software (Düsseldorf, Germany) (selecting two-tailed $t$-test, difference between two dependent means [matched pairs], and a priori power analysis), by fixing a power of 0.95 and a significance level of 0.05 . Statistical analyses were performed using GraphPad Prism version 6.0 (GraphPad Software Inc, La Jolla, CA, USA). Each sample was run in triplicate. Data were expressed as mean $\pm \mathrm{SD}$.

Specific pathway-mediated CEC and CLC values at baseline were compared within treatment groups by using the two-tailed Student's $t$-test for paired samples, after verifying equal variance with the $F$-test and normal distribution of the parameters with the D'Agostino and Pearson omnibus normality test. Significant differences were defined as $p<0.05$.

\section{Results}

\section{Patients characteristics}

The 23 patients enrolled for the study displayed a mean age of $55.39 \pm 12.7$ years and $56.5 \%$ of them were males. Baseline demographic and characteristics of the study population are reported in Table 1. Overall, subjects had clinical and laboratory parameters within a range of normality except

Table I Baseline subjects' characteristics

\begin{tabular}{ll}
\hline Characteristics & $\mathbf{N}=\mathbf{2 3}$ \\
\hline Age $($ years $)$ & $55.39 \pm 12.7$ \\
Male sex $(\mathrm{n}, \%)$ & $13(56.5 \%)$ \\
Body max index $\left(\mathrm{kg} / \mathrm{m}^{2}\right)$ & $27.67 \pm 3.5$ \\
FPG $(\mathrm{mg} / \mathrm{dL})$ & $89.72 \pm 13.9$ \\
SBP $(\mathrm{mmHg})$ & $129.68 \pm 10.9$ \\
DBP $(\mathrm{mmHg})$ & $81.59 \pm 7.1$ \\
TC $(\mathrm{mg} / \mathrm{dL})$ & $218 \pm 36.2$ \\
LDL-C $(\mathrm{mg} / \mathrm{dL})$ & $143 \pm 33.0$ \\
HDL-C $(\mathrm{mg} / \mathrm{dL})$ & $50 \pm 10.8$ \\
TG $(\mathrm{mg} / \mathrm{dL})$ & $125 \pm 62.4$ \\
\hline
\end{tabular}

Note: Data are presented as mean \pm SD or $\mathrm{n}(\%)$.

Abbreviations: FPG, fasting plasma glucose; SBP, systolic blood pressure; DBP, diastolic blood pressure; TC, total cholesterol; LDL-C, low-density lipoprotein cholesterol; HDL-C, high-density lipoprotein cholesterol; TG, triglycerides. 
Table 2 Effect of NUT combination on serum lipid profile

\begin{tabular}{lllll}
\hline & $\begin{array}{l}\text { Baseline } \\
(\mathbf{n}=\mathbf{2 3})\end{array}$ & $\begin{array}{l}\text { NUT } \\
\text { combination } \\
(\mathbf{n}=\mathbf{2 3})\end{array}$ & $\begin{array}{l}\text { Mean } \\
\text { change \% }\end{array}$ & p-value \\
\hline TC $(\mathrm{mg} / \mathrm{dL})$ & $218 \pm 36.2$ & $198 \pm 31.1$ & -9.2 & $<0.001$ \\
LDL-C $(\mathrm{mg} / \mathrm{dL})$ & $143 \pm 33.0$ & $125 \pm 23.1$ & -12.6 & $<0.0 \mid$ \\
$\mathrm{HDL}-\mathrm{I}(\mathrm{mg} / \mathrm{dL})$ & $50 \pm 10.8$ & $49 \pm 11.1$ & -2 & $\mathrm{NS}$ \\
$\mathrm{TG}(\mathrm{mg} / \mathrm{dL})$ & $125 \pm 62.4$ & $116 \pm 58.5$ & -7.2 & $\mathrm{NS}$ \\
apoA-I $(\mathrm{mg} / \mathrm{dL})$ & $128.68 \pm 39.68$ & $131.39 \pm 45.44$ & -2 & $\mathrm{NS}$ \\
\hline
\end{tabular}

Notes: Data are presented as mean \pm SD. Statistical significance was calculated by the two-tailed Student's paired $t$-test.

Abbreviations: TC, total cholesterol; LDL-C, low-density lipoprotein cholesterol; HDL-C, high-density lipoprotein cholesterol; TG, triglycerides; apoA-l, apolipoprotein A-l; NS, not significant; NUT, nutraceutical.

for a slight increase of TC and LDL-C, according to the inclusion criteria.

Table 2 shows the lipid profile at baseline and after treatment with the NUT combination. The NUT combination significantly reduced the plasma level of TC and of LDL-C of about $9.2 \%$ and $12.6 \%$, respectively.

Conversely, no differences were observed after treatment on plasma HDL-C, triglycerides levels and plasma remnants cholesterol calculated by subtracting LDL-C and HDL-C from $\mathrm{TC}^{31}(25 \pm 12.5 \mathrm{mg} / \mathrm{dL}$ before treatment vs $23 \pm 11.7 \mathrm{mg} /$ $\mathrm{dL}$ after treatment; not significant [ns]).

apoA-I concentration was measured in serum samples after precipitation of apoB- containing lipoproteins with polyethylene glycol. The NUT combination did not show any significant effect on apoA-I concentrations.

\section{HDL-CEC}

First, we evaluated the effect of the NUT combination on total HDL-CEC of sera from the enrolled subjects in a model of cAMP-stimulated J774 macrophages. In these conditions, release of cholesterol from cells mainly occurs through $\mathrm{AD}$ and the $\mathrm{ABCA1}$-mediated processes. Compared to baseline, the NUT combination increased total HDL-CEC by an average of $16.1 \%$ (Figure 1A), improving it in $83 \%$ of the patients. Since the major contributions to total HDLCEC are given by $\mathrm{AD}$ and $\mathrm{ABCA1}$, we analyzed these two cholesterol efflux pathways individually. We found that the NUT combination significantly increased HDL-CEC via both $\mathrm{ABCA} 1$ and $\mathrm{AD}$, with $\mathrm{ABCA} 1$ being increased at a higher extent compared to $\mathrm{AD}(+28.5 \%$ and $+8.1 \%$, respectively) (Figure 1B and C).
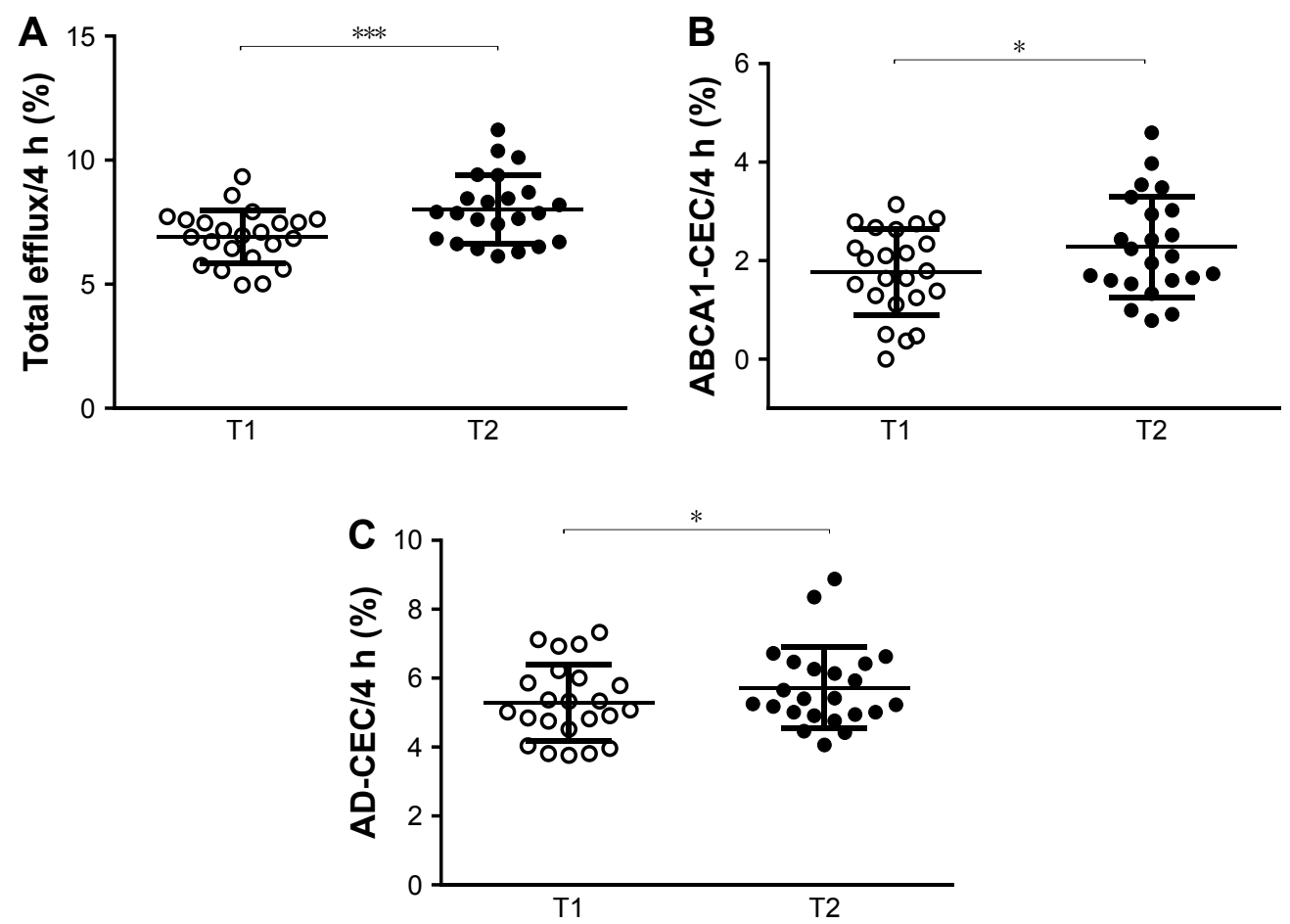

Figure I Effect of NUT combination on serum HDL-CEC.

Notes: Total serum HDL-CEC $(\mathbf{A})$, serum HDL-CEC mediated by ABCAI (B) and by AD $(\mathbf{C})$ in subjects ( $\mathrm{n}=23$ ) at baseline (TI, empty circles) and after (T2, black circles) consumption of the NUT combination. Each point represents the mean of values obtained from evaluation in triplicate of each serum sample. Horizontal lines represent the mean $( \pm S D)$ of values within each subject group. Statistical significance was calculated by the two-tailed Student's paired $t$-test. * $p<0.05$; **** $p<0.00$ I.

Abbreviations: CEC, cholesterol efflux capacity; HDL, high-density lipoproteins; ABCAI, ATP-binding cassette transporter AI; AD, aqueous diffusion; NUT, nutraceutical. 
When we evaluated HDL-CEC mediated by ABCG1, we could not observe any significant changes induced by the NUT combination (before treatment $5.43 \% \pm 2.21 \%$, after treatment $5.07 \% \pm 2.30 \%$; $p=\mathrm{ns}$ ). CEC values for all the analyzed pathways did not correlate with plasma HDL-C levels neither before nor after the consumption of the NUT combination (data not shown).

\section{Serum CLC}

Cell cholesterol content is the result of both cholesterol efflux and influx. ${ }^{32}$ To study the overall serum ability to modulate cellular cholesterol content, we evaluated the effect of the NUT combination on serum CLC in THP-1-derived human macrophages. As shown in Figure 2, the NUT combination significantly reduced serum capacity of loading cells with cholesterol (from $32.15 \pm 7.28$ to $28.47 \pm 6.32 \mu \mathrm{g}$ of cholesterol $/ \mathrm{mg}$ protein, $p<0.05$ ). No correlation was found between CLC values and neither plasma LDL-C levels nor plasma remnants cholesterol, both before and after the NUT combination (data not shown).

\section{Effect of NUT combination on circulating levels of PCSK9}

Since different components of the NUT combination are predicted to have opposite effects on PCSK9, berberine should decrease while monacolin K should increase PCSK9 plasma levels, we measured the PCSK9 serum concentrations

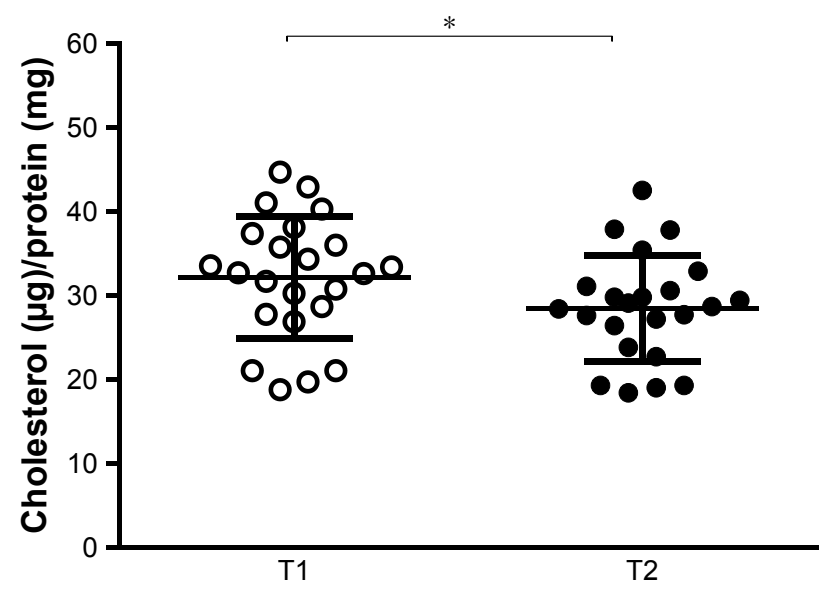

Figure 2 Effect of NUT combination on serum CLC.

Notes: CLC of serum from subjects $(n=23)$ at baseline (TI, empty circles) and after (T2, black circles) consumption of the NUT combination. Each point represents the mean of the values obtained from evaluation in triplicate of each serum sample. Horizontal lines represent the mean $( \pm S D)$ of values within each subject group. Statistical significance was calculated by the two-tailed Student's paired $t$-test. $* p<0.05$.

Abbreviations: NUT, nutraceutical; CLC, cholesterol loading capacity.

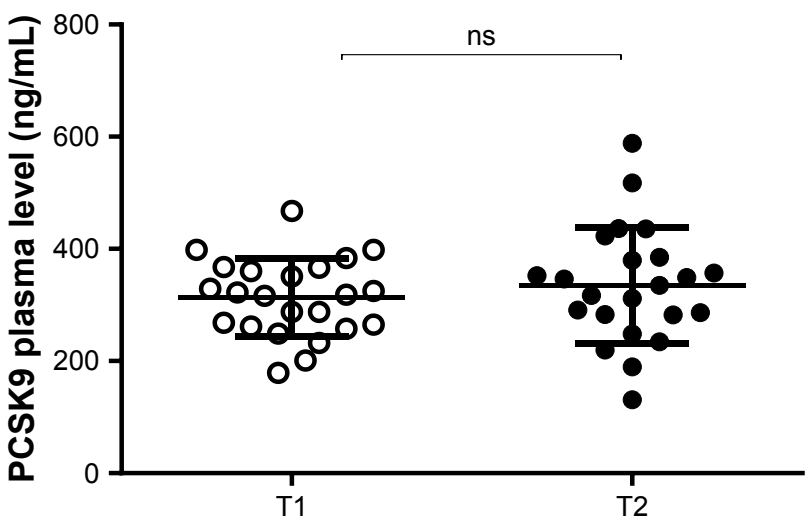

Figure 3 Effect of NUT combination on plasma levels of PCSK9.

Notes: PCSK 9 levels of serum from subjects $(n=23)$ at baseline (TI, empty circles) and after (T2, black circles) consumption of the NUT combination. Each point represents the mean of the values obtained from evaluation in triplicate of each serum sample. Horizontal lines represent the mean $( \pm S D)$ of values within each subject group. Statistical significance was calculated by the two-tailed Student's paired $t$-test.

Abbreviations: NUT, nutraceutical; PCSK9, proprotein convertase subtilisin kexin type 9 (PCSK9); ns, not significant.

by ELISA assay. The NUT combination did not show any significant effect on PCSK9 levels $(312.9 \pm 69.4$ and $334.8 \pm 103.5 \mathrm{ng} / \mathrm{mL}$, respectively; $p=\mathrm{ns}$ ) (Figure 3).

\section{Discussion}

In this study, we investigated the effect of a novel NUT combination of berberine, monacolin K, and M. alba extract $\left(\right.$ LopiGLIK $^{\circledR}$ ) on serum lipid-related factors involved in the protective process of reverse cholesterol transport. Serum CEC, serum CLC and serum level of PCSK9 represent factors that may directly modulate cholesterol homeostasis in macrophages, the main cellular component of atheroma. ${ }^{33}$ The evaluation of these parameters provides a serum functional lipid profile related to $\mathrm{CV}$ risk independent of serum lipid concentrations.

Consistent with a previous study, ${ }^{25}$ our results confirm that the NUT combination favorably modifies the plasma lipid profile in terms of reduction of TC and LDL-C. In the present study, the most striking result is that, despite the lack of an effect on HDL-C plasma level, the NUT combination improves serum HDL-CEC, the main antiatherogenic property of these lipoproteins. Thus, the NUT combination appears to improve HDL functionality without altering plasma HDL-C concentrations. Only few studies have previously shown a positive effect of NUTs on HDL-CEC. ${ }^{22,34}$ Our NUT combination increased total HDL-CEC, the cholesterol efflux pathway recently identified as a $\mathrm{CV}$ risk biomarker and a promising target for the prevention and therapies of $\mathrm{CV}$ diseases, independently of plasma HDL-C concentrations. ${ }^{4-6}$ 
When we analyzed the individual pathways contributing to total efflux, $\mathrm{ABCA} 1$ and $\mathrm{AD}$, we found that the increase of total CEC was mainly related to the improved process of ABCA1-mediated CEC. This cholesterol efflux pathway represents the main route by which macrophages release cholesterol to serum and oppose foam cell formation and atherosclerosis progression..$^{35}$ The improvement of the ABCA1-mediated CEC, without an effect on ABCG1-mediated CEC, suggests a shift of HDL particles distribution toward lipid-poor pre- $\beta$ HDL specific for the ABCA1 pathway. ${ }^{36,37}$ Consistent with our results, the increase in pre- $\beta$ HDL may occur independently of serum HDL-C. ${ }^{36}$ In addition, when we measured apoA-I levels in apoB-depleted serum, we found that the NUT combination did not affect even apoA-I concentrations. Our finding can be explained by the fact that neither apoA-I nor HDL-C serum concentration values reflect the actual serum content of pre- $\beta$ HDL, the specific HDL particles acting as acceptor for ABCA1. ${ }^{27,38}$ Moreover, free apoA-I active as ABCA1 acceptor constitutes only about $5 \%$ of the circulating apoA-I. ${ }^{39}$

It may be speculated that the increase in pre- $\beta$ HDL could be related to a NUT-induced production of these nascent particles that may involve the stimulation of hepatic ABCA1 transporter. ${ }^{40}$ This NUT effect might be related to the presence of monacolin K, given the documented stimulating effect of statins on hepatic ABCA1 both in vitro and in vivo. ${ }^{41,42}$ However, the impact of statin therapy on HDLCEC does not appear to be substantial, in agreement with the modest effect of statins on HDL-C concentration and on HDL subclasses distribution ${ }^{43}$ and no data on CEC are available for berberine and $M$. alba. A possible effect by berberine and M. alba on HDL-CEC cannot be excluded, although no literature is available yet in this regard.

The other serum lipoprotein function that we evaluated, CLC, is the ability of serum to load macrophages with cholesterol. Treatment with the NUT combination reduced this pro-atherogenic potential of serum. Consistent with previous observations, ${ }^{11}$ this effect did not correlate to the reduction of plasma LDL-C concentrations. Beyond LDL-C, also remnant cholesterol which includes VLDL, namely non-LDL and nonHDL-C, ${ }^{31}$ contributes to macrophage cholesterol loading. ${ }^{44}$ However, we did not observe any changes in remnants levels. Most probably, the observed reduced serum CLC is related to the combined effect of both decrease on LDL levels and the increase of HDL-CEC induced by the NUT combination treatment.

A recent study demonstrated that circulating PCSK9 was associated to carotid atherosclerosis independent of the effect on LDL-C plasma level. ${ }^{15}$ More recently, we demonstrated that PCSK9 in macrophages directly inhibits the efflux of cholesterol specifically mediated by ABCA1. ${ }^{17}$ This effect would counteract the beneficial increase in ABCA1-mediated CEC induced by our NUT combination. We therefore evaluated if our present NUT approach could modulate serum functionality not only in terms of CEC and CLC, but also of plasma PCSK9 concentrations.

In contrast to the results reported with monacolin $\mathrm{K}$ alone, ${ }^{46}$ our results indicate that our NUT combination did not modify PCSK9 plasma levels. We may speculate that the effect of monacolin K, the statin produced by Monascus purpureus present in our NUT combination, toward an increase of plasma PCSK $9,{ }^{46}$ may be counteracted by the presence of berberine, a known negative modulator of PCSK9 concentration in plasma. ${ }^{45,47}$ In addition, extracts from dried immature M. alba fruits have been recently shown to have an inhibitory activity on PCSK $9 .{ }^{48}$

This result indicates an additional potential benefit of a NUT combination compared to the statin single administration.

\section{Limitations of the study}

The small number of patients in this study, with a relatively wide range of age, limited the potential for detailed statistical analyses, but it provides a picture of a real-life population. In addition, our study population was large enough to meet the required statistical power to demonstrate the differences in both HDLCEC and serum CLC. It is worth pointing out that the variation of the measured parameters was consistently observed in $\sim 80 \%$ of the examined subjects. As this is the first study evaluating the effect of a NUT combination on lipoprotein functions, despite the modest effect, it provides an important direction for further confirmatory studies in a larger group of subjects.

\section{Conclusion}

Our results demonstrate that, despite the lack of effect on HDL plasma concentrations, our NUT combination may improve lipoprotein protective functions providing complementary beneficial effects for $\mathrm{CV}$ risk prevention, particularly useful in moderate dyslipidemic subjects.

\section{Acknowledgment}

The authors acknowledge Akademy Pharma Srl (Milano, Italy) for supporting this study.

\section{Author contributions}

All authors have substantially contributed to the manuscript and approved the final article. FB conceived the study; VT, FR, and RI recruited patients; MPA, FZ, and SM performed the experiments and acquired data; and MPA, NF, and FZ analyzed 
and interpreted the data. The article was drafted by MPA, FZ, and NF and was critically revised by all authors. All authors contributed toward data analysis, drafting and revising the paper and agree to be accountable for all aspects of the work.

\section{Disclosure}

Franco Bernini received support for this study from Akademy Pharma Srl (Milano, Italy). The other authors report no conflicts of interest in this work.

\section{References}

1. Catapano AL, Graham I, De Backer G, et al. 2016 ESC/EAS guidelines for the management of dyslipidaemias. Eur Heart J. 2016;37: 2999-3058.

2. Barrios V, Escobar C, Cicero AF, et al. A nutraceutical approach (Armolipid Plus) to reduce total and LDL cholesterol in individuals with mild to moderate dyslipidemia: review of the clinical evidence. Atheroscler Suppl. 2017;24:1-15.

3. Sahebkar A, Serban MC, Gluba-Brzozka A, et al. Lipid-modifying effects of nutraceuticals: an evidence-based approach. Nutrition. 2016;32:1179-1192.

4. Anastasius M, Kockx M, Jessup W, Sullivan D, Rye KA, Kritharides L. Cholesterol efflux capacity: an introduction for clinicians. Am Heart J 2016;180:54-63.

5. Khera AV, Cuchel M, de la Llera-Moya M, et al. Cholesterol efflux capacity, high-density lipoprotein function, and atherosclerosis. $N$ Engl J Med. 2011;364:127-135.

6. Rohatgi A, Khera A, Berry JD, et al. HDL cholesterol efflux capacity and incident cardiovascular events. N Engl J Med. 2014;371: 2383-2393.

7. Saleheen D, Scott R, Javad S, et al. Association of HDL cholesterol efflux capacity with incident coronary heart disease events: a prospective case-control study. Lancet Diabetes Endocrinol. 2015;3: 507-513

8. Mody P, Joshi PH, Khera A, Ayers CR, Rohatgi A. Beyond coronary calcification, family history, and C-reactive protein: cholesterol efflux capacity and cardiovascular risk prediction. J Am Coll Cardiol. 2016; 67:2480-2487.

9. Weibel GL, Drazul-Schrader D, Shivers DK, et al. Importance of evaluating cell cholesterol influx with efflux in determining the impact of human serum on cholesterol metabolism and atherosclerosis. Arterioscler Thromb Vasc Biol. 2014;34:17-25.

10. Adorni MP, Zimetti F, Puntoni M, et al. Cellular cholesterol efflux and cholesterol loading capacity of serum: effects of LDL-apheresis. J Lipid Res. 2012;53:984-989.

11. Ronda N, Greco D, Adorni MP, et al. Newly identified antiatherosclerotic activity of methotrexate and adalimumab: complementary effects on lipoprotein function and macrophage cholesterol metabolism. Arthritis Rheumatol. 2015;67(5):1155-1164.

12. Norata GD, Tavori H, Pirillo A, Fazio S, Catapano AL. Biology of proprotein convertase subtilisin kexin 9: beyond low-density lipoprotein cholesterol lowering. Cardiovasc Res. 2016;112:429-442.

13. Ferri N, Marchiano S, Tibolla G, et al. PCSK9 knock-out mice are protected from neointimal formation in response to perivascular carotid collar placement. Atherosclerosis. 2016;253:214-224.

14. Leander K, Malarstig A, Van't Hooft FM, et al. Circulating proprotein convertase subtilisin/kexin type 9 (PCSK9) predicts future risk of cardiovascular events independently of established risk factors. Circulation. 2016;133:1230-1239.

15. Xie W, Liu J, Wang W, et al. Association between plasma PCSK9 levels and 10-year progression of carotid atherosclerosis beyond LDL-C: a cohort study. Int J Cardiol. 2016;215:293-298.
16. Ruscica M, Ferri N, Fogacci F, et al. Circulating levels of proprotein convertase subtilisin/kexin type 9 and arterial stiffness in a large population sample: data from the Brisighella Heart Study. J Am Heart Assoc. Epub 2017 May 3.

17. Adorni MP, Cipollari E, Favari E, et al. Inhibitory effect of PCSK9 on ABCA1 protein expression and cholesterol efflux in macrophages. Atherosclerosis. 2017;256:1-6.

18. Feig JE, Hewing B, Smith JD, Hazen SL, Fisher EA. High-density lipoprotein and atherosclerosis regression: evidence from preclinical and clinical studies. Circ Res. 2014;114:205-213.

19. Rizzo M, Berneis K, Koulouris S, et al. Should we measure routinely oxidised and atherogenic dense low-density lipoproteins in subjects with type 2 diabetes? Int J Clin Pract. 2010;64:1632-1642.

20. Richard C, Couture P, Desroches S, et al. Effect of the Mediterranean diet with and without weight loss on surrogate markers of cholesterol homeostasis in men with the metabolic syndrome. Br J Nutr. 2012; 107:705-711.

21. Pu S, Rodriguez-Perez C, Ramprasath VR, Segura-Carretero A, Jones PJ. Dietary high oleic canola oil supplemented with docosahexaenoic acid attenuates plasma proprotein convertase subtilisin kexin type 9 (PCSK9) levels in participants with cardiovascular disease risk: a randomized control trial. Vascul Pharmacol. 2016;87:60-65.

22. Zhu Y, Huang X, Zhang Y, et al. Anthocyanin supplementation improves HDL-associated paraoxonase 1 activity and enhances cholesterol efflux capacity in subjects with hypercholesterolemia. J Clin Endocrinol Metab. 2014;99:561-569.

23. Badeau R, Jauhiainen M, Metso J, et al. Effect of isolated isoflavone supplementation on ABCA1-dependent cholesterol efflux potential in postmenopausal women. Menopause. 2007;14:293-299.

24. Momtazi AA, Banach M, Pirro M, Katsiki N, Sahebkar A. Regulation of PCSK9 by nutraceuticals. Pharmacol Res. 2017;120:157-169.

25. Trimarco V, Izzo R, Stabile E, et al. Effects of a new combination of nutraceuticals with Morus alba on lipid profile, insulin sensitivity and endothelial function in dyslipidemic subjects. A cross-over, randomized, double-blind trial. High Blood Press Cardiovasc Prev. 2015;22: $149-154$.

26. Khalil H, Murrin C, O'Reilly M, et al. Total HDL cholesterol efflux capacity in healthy children - associations with adiposity and dietary intakes of mother and child. Nutr Metab Cardiovasc Dis. 2017;27: $70-77$.

27. Favari E, Ronda N, Adorni MP, et al. ABCA1-dependent serum cholesterol efflux capacity inversely correlates with pulse wave velocity in healthy subjects. J Lipid Res. 2013;54:238-243.

28. Pisciotta L, Favari E, Magnolo L, et al. Characterization of three kindreds with familial combined hypolipidemia caused by loss-offunction mutations of ANGPTL3. Circ Cardiovasc Genet. 2012;5: 42-50.

29. Markwell MA, Haas SM, Bieber LL, Tolbert NE. A modification of the Lowry procedure to simplify protein determination in membrane and lipoprotein samples. Anal Biochem. 1978;87:206-210.

30. Ruscica M, Ferri N, Macchi C, et al. Liver fat accumulation is associated with circulating PCSK9. Ann Med. 2016;48:384-391.

31. Varbo A, Nordestgaard BG. Remnant lipoproteins. Curr Opin Lipidol. 2017;28:300-307.

32. Zimetti F, Weibel GK, Duong M, Rothblat GH. Measurement of cholesterol bidirectional flux between cells and lipoproteins. J Lipid Res. 2006;47:605-613.

33. Yamamoto S, Narita I, Kotani K. The macrophage and its related cholesterol efflux as a HDL function index in atherosclerosis. Clin Chim Acta. 2016;457:117-122.

34. Hernaez A, Fernandez-Castillejo S, Farras M, et al. Olive oil polyphenols enhance high-density lipoprotein function in humans: a randomized controlled trial. Arterioscler Thromb Vasc Biol. 2014;34: 2115-2119.

35. Adorni MP, Zimetti F, Billheimer JT, et al. The roles of different pathways in the release of cholesterol from macrophages. J Lipid Res. 2007;48:2453-2462. 
36. de la Llera-Moya M, Drazul-Schrader D, Asztalos BF, Cuchel M, Rader DJ, Rothblat GH. The ability to promote efflux via ABCA1 determines the capacity of serum specimens with similar high-density lipoprotein cholesterol to remove cholesterol from macrophages. Arterioscler Thromb Vasc Biol. 2010;30:796-801.

37. Favari E, Lee M, Calabresi L, et al. Depletion of pre-beta-high density lipoprotein by human chymase impairs ATP-binding cassette transporter A1- but not scavenger receptor class B type I-mediated lipid efflux to high density lipoprotein. J Biol Chem. 2004;279:9930-9936.

38. Ronda N, Favari E, Borghi MO, et al. Impaired serum cholesterol efflux capacity in rheumatoid arthritis and systemic lupus erythematosus. Ann Rheum Dis. 2014;73:609-615.

39. Duong PT, Weibel GL, Lund-Katz S, Rothblat GH, Phillips MC. Characterization and properties of pre beta-HDL particles formed by ABCA1-mediated cellular lipid efflux to apoA-I. J Lipid Res. 2008; 49:1006-1014.

40. Lee JY, Parks JS. ATP-binding cassette transporter AI and its role in HDL formation. Curr Opin Lipidol. 2005;16:19-25.

41. Kobayashi M, Gouda K, Chisaki I, Ochiai M, Itagaki S, Iseki K. Regulation mechanism of ABCA1 expression by statins in hepatocytes. Eur J Pharmacol. 2011;662:9-14.

42. Song G, Liu J, Zhao Z, et al. Simvastatin reduces atherogenesis and promotes the expression of hepatic genes associated with reverse cholesterol transport in apoE-knockout mice fed high-fat diet. Lipids Health Dis. 2011;10:8.
43. Gomaraschi M, Adorni MP, Banach M, Bernini F, Franceschini G, Calabresi L. Effects of established hypolipidemic drugs on HDL concentration, subclass distribution, and function. Handb Exp Pharmacol. 2015;224:593-615.

44. Bernelot Moens SJ, Verweij SL, Schnitzler JG, et al. Remnant cholesterol elicits arterial wall inflammation and a multilevel cellular immune response in humans. Arterioscler Thromb Vasc Biol. 2017;37: 969-975.

45. Pirillo A, Catapano AL. Berberine, a plant alkaloid with lipid- and glucose-lowering properties: from in vitro evidence to clinical studies. Atherosclerosis. 2015;243:449-461.

46. Sahebkar A, Simental-Mendia LE, Guerrero-Romero F, Golledge J, Watts GF. Effect of statin therapy on plasma proprotein convertase subtilisin kexin 9 (PCSK9) concentrations: a systematic review and meta-analysis of clinical trials. Diabetes Obes Metab. 2015;17: 1042-1055.

47. Cameron J, Ranheim T, Kulseth MA, Leren TP, Berge KE. Berberine decreases PCSK9 expression in HepG2 cells. Atherosclerosis. 2008; 201:266-273.

48. Pel P, Chae HS, Nhoek P, Kim YM, Chin YW. Chemical constituents with proprotein convertase subtilisin/kexin type 9 mRNA expression inhibitory activity from dried immature Morus alba fruits. J Agric Food Chem. 2017;65:5316-5321.
Therapeutics and Clinical Risk Management

\section{Publish your work in this journal}

Therapeutics and Clinical Risk Management is an international, peerreviewed journal of clinical therapeutics and risk management, focusing on concise rapid reporting of clinical studies in all therapeutic areas, outcomes, safety, and programs for the effective, safe, and sustained use of medicines. This journal is indexed on PubMed Central, CAS,

\section{Dovepress}

EMBase, Scopus and the Elsevier Bibliographic databases. The manuscript management system is completely online and includes a very quick and fair peer-review system, which is all easy to use. Visit http://www.dovepress.com/testimonials.php to read real quotes from published authors. 